\title{
Influencia de las condiciones de carga en la generación de úlceras por presión internas en amputados transfemorales
}

\section{Influence of load conditions for deep tissue injury development in transfemoral amputees}

\author{
Carlos Mejía-Blandón ${ }^{1}$, Liliana Bustamante-Goez ${ }^{2}$, Junes Villarraga-Ossa ${ }^{3}$ \\ ${ }^{1}$ Grupo de Diseño Mecánico-GDM, Ingeniería Mecánica, Universidad de Antioquia, Colombia. \\ Email: carlos.mejiab@udea.edu.co \\ ${ }^{2}$ Grupo de Diseño Mecánico-GDM, Universidad de Antioquia, Colombia. Email: liliana.bustamante@udea.edu.co \\ ${ }^{3}$ Grupo de Diseño Mecánico-GDM, Universidad de Antioquia, Colombia. Email: junes.villarraga@udea.edu.co
}

RECIBIDO: Marzo 10, 2017. ACEPTADO: Julio 29, 2017. VERSIÓN FINAL: Octubre 12, 2017.

\section{RESUMEN}

En esta investigación se realiza la modelación tridimensional del miembro residual de un amputado transfemoral que incluye la piel, el tejido graso, el músculo y el fémur residual. Se establecen las condiciones de carga para la fase de apoyo durante el ciclo de la marcha normal, la bipedestación y la marcha por escaleras de un amputado transfemoral de $88 \mathrm{~kg}$ y una estatura de $1.7 \mathrm{~m}$. Se realizó un análisis de la incidencia de las condiciones de carga sobre los esfuerzos y deformaciones en el tejido muscular, para establecer su influencia sobre la generación de úlceras por presión internas en el miembro residual del amputado. Del análisis se obtuvo que el esfuerzo a compresión promedio más alto es cercano a $18.6 \mathrm{kPa}$ y la deformación promedio más alta a compresión es de $56.8 \%$, esto sucede cuando se realiza marcha por escaleras o superficies inclinadas. La mayor concentración de esfuerzo y deformación se presenta en la zona distal del fémur residual en el tejido muscular.

PALABRAS CLAVE: Úlceras por presión; úlceras por presión internas; UP; UPI; amputado transfemoral; elementos finitos; EF; biomecánica.

\begin{abstract}
In this research, three-dimensional modeling of the residual limb of a transfemoral amputee is performed, which includes skin, fat tissue, muscle and residual femur. The loading conditions are established for the support phase during the normal gait cycle, the standing and walking through stairs of a transfemoral amputee of $88 \mathrm{~kg}$ and a height of 1.7 $\mathrm{m}$. An analysis of the incidence of load conditions on the stresses and deformations in muscle tissue was made to establish its influence on the development of deep tissue injuries (DTI) in the residual limb of the amputee. From the analysis it was obtained that the average compressive stress is close to $18.6 \mathrm{kPa}$ and the highest average strain at compression is $56.8 \%$, it happens when the individual is walking through stairs or slope. The maximum concentration of stress and strain occurs in the distal area of the residual femur in muscle tissue.
\end{abstract}

KEYWORDS: Pressure ulcers; deep pressure ulcers; deep tissue injury; PU; DPU; transfemoral amputation; finite element; FE; biomechanics. 


\section{INTRODUCCIÓN}

Las personas con movilidad reducida están propensos a padecer heridas internas o superficiales, conocidas como úlceras por presión (UP); en el caso de las heridas superficiales su detección es notoria y se pueden tratar con mayor facilidad, cuando se trata de heridas internas el avance puede ser progresivo y silencioso de tal forma que cuando son detectadas tienen un estado avanzado y de difícil tratamiento. Las UP se caracterizan por ser lesiones que afectan las células de los tejidos blandos debido a las cargas combinadas que se aplican en estos. En personas con amputación transfemoral el proceso de calzado de la prótesis y el ciclo de la marcha generan esfuerzos en zonas de la dermis que están en contacto con la prótesis, a su vez las reacciones generadas se transmiten a los tejidos internos provocando esfuerzos y deformaciones que se reflejan en la zona distal del fémur residual y el músculo esquelético que está próximo al hueso. Por las reacciones desarrolladas es posible que se puedan generar UP superficiales en las zonas de la piel que están en contacto con la prótesis y UP internas en las zonas musculares que están próximas a las prominencias óseas.

Las presiones admisibles y la aparición de las UP son función de las características propias de cada individuo [1], [2]. Hay una serie de factores que pueden contribuir al daño en el tejido, como es el caso de la exposición a cargas prolongadas, que pueden disminuir la capacidad del organismo de transportar nutrientes y oxígeno a los tejidos, produciendo así la muerte celular [3]. Se ha notado que el factor principal que influye en la generación de las heridas internas es la presencia de esfuerzos cortantes excesivos en los tejidos. Las UP se desarrollan en los tejidos blandos (piel y músculo), la evolución de las heridas varía de un individuo a otro [4]. Es posible hacer una determinación de la relación que hay entre las cargas aplicadas y las deformaciones inducidas por las cargas aplicadas, donde se muestra que un aumento en las cargas refleja un aumento en las deformaciones presentes en el tejido y que los esfuerzos por compresión y las deformaciones que se presentan por los desplazamientos, se ven reflejadas directamente en el tejido muscular. El umbral de esfuerzos y deformaciones que afectan las células del tejido muscular no está definido en personas con amputación, sin embargo en estudios realizados por Gefen et al [5], las UP internas se pueden generar en el tejido interno cuando se encuentra bajo la acción de presiones que están por encima del umbral de muerte celular de $32 \mathrm{kPa}$ en un lapso de tiempo de una hora, para células de tejido muscular animal, y cuando se presentan deformaciones de $65 \%$ por tiempos de exposición mayores a una hora las células de un tejido muscular artificial se pueden ver afectadas [6].
El nivel de esfuerzo que se puede soportar sin que se presente un alto riesgo de desarrollar UP está determinado por el tiempo que permanezca el paciente bajo los efectos de las cargas.

En esta investigación se busca desarrollar estrategias de prevención, para evitar la generación de heridas internas (UPIs) en amputados transfemorales, por tal razón, se realizó un análisis por elementos finitos del miembro residual de un amputado transfemoral para establecer la tendencia y las zonas de concentración de esfuerzos y deformaciones, que pueden causar úlceras por presión internas en el muñón del paciente. El modelo tridimensional incluyó la piel, el tejido graso, el músculo, el fémur residual y el socket de la prótesis. Para determinar los esfuerzos y deformaciones en el muñón se tuvo en cuenta el proceso de calzado de la prótesis y las cargas generadas en la fase de apoyo de la marcha normal, bipedestación y monopedastación por escaleras o superficies inclinadas.

Los resultados de esta investigación pueden ser útiles para apoyar las estrategias de prevención de las UPIs en pacientes que sufren de cuadriplejía o paraplejía, que permanecen postradas en una cama o en una silla de ruedas.

\section{MÉTODOS}

Para determinar los esfuerzos y deformaciones en el muñón causados por las condiciones de carga, se tomó como referencia el modelo multicapa de un muñón con espesor de tejido adiposo de $10 \mathrm{~mm}$ y longitud del miembro residual de $23 \mathrm{~cm}$ de un paciente con amputación transfemoral de $1.7 \mathrm{~m}$ de estatura y una masa de $88 \mathrm{~kg}$. Las condiciones de carga aplicadas fueron las generadas durante la fase de apoyo de la marcha, el apoyo en bipedestación, monopedestación por escaleras o superficies inclinadas y el calzado de la prótesis. Se emplearon modelos hiperelásticos para modelar los tejidos blandos y modelos elásticos para los demás elementos. El modelado y análisis se llevó a cabo de acuerdo a los siguientes pasos:

\subsection{Datos antropométricos}

Para la desarrollo del estudio se construye un modelo genérico del muñón de un amputado transfemoral, partiendo de datos encontrados en diferentes estudios antropométricos donde se establecen las dimensiones de las extremidades del cuerpo [7]. El espesor de tejido adiposo y la longitud del miembro residual son $10 \mathrm{~mm}$ y $23 \mathrm{~cm}$ respectivamente de acuerdo en lo obtenido en [8]. 


\subsection{Modelos CAD del muñón y el socket}

Con el objetivo de obtener la distribución y magnitud de los esfuerzos y deformaciones que se desarrollan en el tejido interno del muñón de un paciente amputado transfemoral, se desarrolla un modelo multicapa del muñón dividido en tres partes: músculo, grasa y piel. El modelo se construye a partir de la información presentada en [7], [9]-[11]. En la Figura 1, se muestra la representación multicapa del modelo.

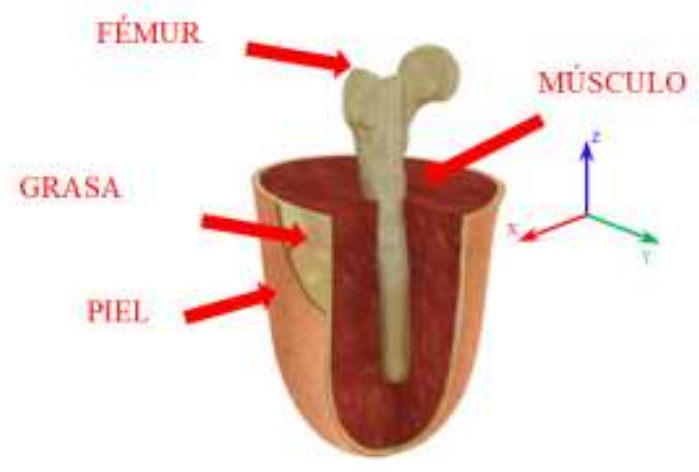

Figura 1. Modelo residual amputado transfemoral. Fuente. Elaboración propia.

La representación del modelo CAD construido se puede ver en la siguiente figura.
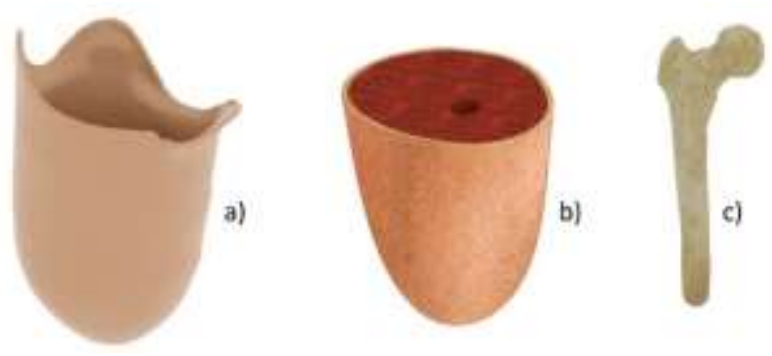

Figura 2. Modelos CAD (a) Socket, (b) Muñón multicapa y (c) Hueso. Fuente. Elaboración propia.

El procedimiento de construcción del modelo se realizó empleando el programa SolidWorks versión 2010.

\subsection{Modelos numéricos interacción socket - muñón}

Una vez con el modelo construido, se procedió a realizar el respectivo modelo de elementos finitos, empleando el software ANSYS versión 14. Se utilizó un modelo explicito debido a las características del sistema, donde se hace la emulación del proceso de calzado del socket, a causa de este proceso se desarrollan estados de esfuerzos y deformaciones iniciales en los tejidos blandos del muñón, posteriormente se aplicaron las reacciones generadas por las diferentes condiciones de carga, de los resultados y esfuerzos que se desarrollan se determinó la influencia del tipo de carga en la generación de úlceras por presión en el tejido muscular.

\subsection{Propiedades de los materiales}

Para las propiedades mecánicas de los materiales que componen el sistema socket - muñón, se asumieron modelos elásticos-lineales para el hueso y el socket como es sugerido por [12]-[14] (Ver Tabla 1). Los tejidos blandos (músculo, grasa y piel) se modelaron como materiales hiperelásticos de acuerdo con [15]-[29]. El comportamiento de los tejidos blandos fue definido mediante la función de energía de deformación de Mooney Rivlin [30] implementada en Ansys versión 14, donde los parámetros constitutivos $\mathrm{C}_{10}, \mathrm{C}_{01}, \mathrm{D}_{1}$ utilizados se muestran en la Tabla 1. Para todos estos se consideró un coeficiente de Poisson $v=0.49$ y se tomó la densidad de la piel como $1100 \mathrm{Kg} / \mathrm{m}^{3}$, la densidad del músculo $1064 \mathrm{Kg} / \mathrm{m}^{3}$ y la densidad del tejido graso como 900 $\mathrm{Kg} / \mathrm{m}^{3}[31]$.

Tabla 1. Propiedades de los materiales.

\begin{tabular}{|l|l|l|l|}
\hline \multicolumn{4}{|l|}{ Materiales elásticos } \\
\hline Material & $\begin{array}{l}\text { Módulo } \\
\text { de Young }\end{array}$ & $\begin{array}{l}\text { Coeficiente } \\
\text { de Poisson }\end{array}$ & Densidad \\
\hline $\begin{array}{l}\text { Socket } \\
{[12],} \\
{[13]}\end{array}$ & $\begin{array}{l}1.5 \\
\mathrm{GPa}\end{array}$ & 0.3 & $\begin{array}{l}800 \\
\mathrm{Kg} / \mathrm{m} 3\end{array}$ \\
\hline $\begin{array}{l}\text { Hueso } \\
{[12],} \\
{[13],} \\
{[32]}\end{array}$ & $\begin{array}{l}15 \\
\mathrm{GPa}\end{array}$ & 0.3 & $\begin{array}{l}2000 \\
\mathrm{Kg} / \mathrm{m} 3\end{array}$ \\
\hline Parámetros constitutivos de los tejidos blandos \\
\hline $\begin{array}{l}\text { Tejido } \\
\text { Blando }\end{array}$ & $\begin{array}{l}\mathrm{C} 10 \\
\text { (kPa) }\end{array}$ & $\mathrm{C} 01(\mathrm{kPa})$ & $\mathrm{D} 1\left(\mathrm{MPa}^{-1}\right)$ \\
\hline Piel & 9.4 & 82 & 0 \\
\hline Grasa & 0.143 & 0 & 70.2 \\
\hline Músculo & 8.075 & 0 & 1.243 \\
\hline
\end{tabular}

Fuente. Elaboración propia.

\subsection{Condiciones de borde}

Se debe definir las condiciones de borde adecuadas que garanticen el comportamiento real del sistema, para el caso de la fijación se utilizó una restricción de movimiento en la cabeza del fémur (ver Figura 3), debido a que en la fase de apoyo de la marcha la restricción de 
desplazamiento se realiza sobre esta parte del hueso en la articulación de la cadera.

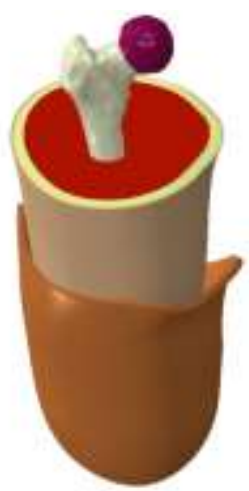

Figura 3. Fijación del hueso. Fuente. Elaboración propia.

En el modelo se consideraron los procesos reales que ocurren en el uso de la prótesis, los cuales incluyeron el calzado de la prótesis y la fase de apoyo de la marcha en su condición más crítica. A partir de esto, la simulación se dividió en tres etapas: la primera que corresponde al desplazamiento que simula la postura del socket; la segunda fue una fase de relajación para lograr disipar la energía cinética inducida en el sistema a raíz del movimiento del socket sobre el muñón y la tercera etapa consistió en la aplicación de la carga correspondiente a la fase de apoyo.

Las interacciones entre los diferentes componentes se definieron como sigue: entre el hueso y el músculo se asignó una condición de bonded, para restringir el desplazamiento entre los dos tejidos, es decir, estas superficies permanecen unidas durante toda la simulación, el mismo tipo de consideración se hizo para las superficies del músculo y la grasa y entre la grasa y la piel, dichas consideraciones se hicieron con el fin de optimizar el tiempo computacional, aunque puede estar un poco alejado del comportamiento real, es utilizada comúnmente por otros investigadores [33]-[36] como una simplificación valida. El contacto entre el socket y el muñón se asumió de tipo frictional, con un coeficiente de fricción $(\mathrm{CF})$ entre el polipropileno y la piel de 0.7, para la interacción entre el socket y la piel en el modelo y que estuviera presente en todas las fases de la simulación, este valor es utilizado en varias investigaciones [30], [37], [38], en las cuales midieron experimentalmente el valor de CF en pacientes amputados transtibiales y el valor del $\mathrm{CF}$ de la piel con diferentes materiales.

\subsubsection{Postura del socket.}

Esta etapa representa el movimiento vertical del socket desde una posición inmediatamente anterior a que empiece el contacto con el muñón hasta una posición final en la que contiene todo el tejido y el hueso en un intervalo de tiempo de diez segundos. Durante el desplazamiento del socket, el tejido blando debe deformarse considerablemente para ser contenido en el encaje, en la Figura 4 se ilustra el proceso de postura del socket.
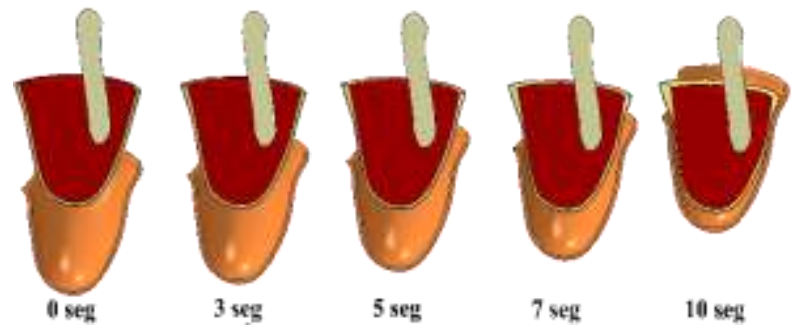

Figura 4. Postura del socket [39].

Una buena sujeción permite lograr el ajuste necesario entre el muñón y el socket en la interacción de la prótesis en la marcha humana, reduciendo el riesgo de sufrir laceraciones por el juego que puede quedar entre el socket y el muñón, por ende la persona puede gozar de mayor confort.

\subsubsection{Relajación.}

La fase de relajación se considera como una fase de transición entre el proceso de postura del socket (fase de desplazamiento) y el momento de apoyo de la marcha (fase de aplicación de la carga). En este paso, se estableció un intervalo de tres segundos para permitir que el tejido blando se ajustara a la geometría del socket, para disipar los cambios que se producían como ondas o pliegues en la piel y para disipar la energía de los tejidos, producto de las grandes deformaciones que se produjeron durante la etapa de desplazamiento.

\subsubsection{Aplicación de carga.}

La carga aplicada al modelo fue la más crítica durante la marcha normal (fase de apoyo), esta carga se localiza en la parte rígida del socket, donde se une por medio de los adaptadores al tubo soporte del muslo de la prótesis. Para el cálculo de las fuerzas sobre el socket, se calcularon las reacciones sobre el talón del pie, empleando las fuerzas reactivas sobre el piso a través del talón del pie, los pesos de cada uno de los componentes de la prótesis y del muñón y las aceleraciones angulares (ver Figura 5). Se tomó como referencia el peso corporal de una persona de $88 \mathrm{~kg}$ y una estatura de $1.7 \mathrm{~m} \mathrm{[7].} \mathrm{La} \mathrm{carga} \mathrm{fue} \mathrm{aplicada}$ después de la fase de relajación durante 0.1 segundos. Para observar la influencia de las condiciones de carga en la generación de UP, se emplearon los valores de carga en los cuales la persona se encontraba apoyada en los dos pies (bipedestación) y cuando realizaba la marcha subiendo $\mathrm{o}$ bajando escaleras $\mathrm{y}$ ascendiendo $\mathrm{o}$ 
descendiendo por superficies inclinadas. En el caso de la bidipedestación las reacciones sobre la prótesis eran la mitad de las de la fase de apoyo de la marcha normal y en los demás casos, las reacciones aumentaron cinco veces. Para el análisis por elementos finitos se aplicaron las reacciones y momentos desarrollados por cada condición de carga como se muestra en la Tabla 2.
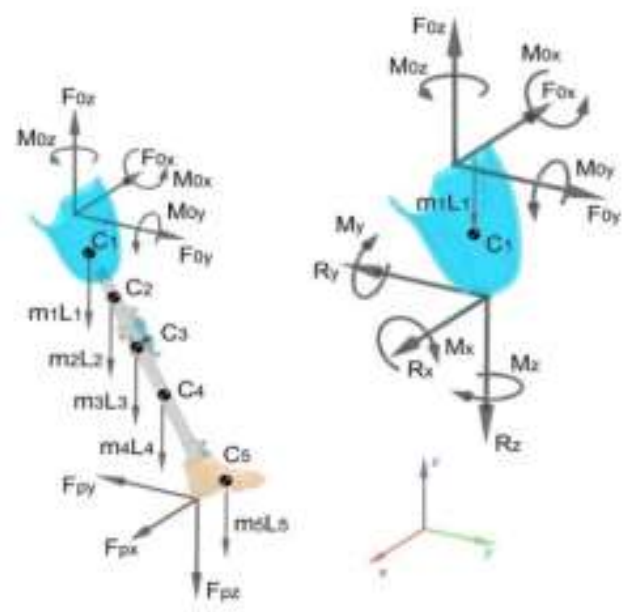

Figura 5. Cinemática inversa. Fuente. Elaboración propia.
Tabla 3. Información del mallado para cada componente.

\begin{tabular}{|c|c|c|c|}
\hline & $\begin{array}{c}\mathbf{N}^{\circ} . \\
\text { elementos }\end{array}$ & $\mathbf{N}^{\circ}$ nodos & $\begin{array}{c}\text { Tamaño } \\
\text { Elemento } \\
{[\mathbf{m m}]}\end{array}$ \\
\hline Hueso & 46532 & 10056 & 6 \\
\hline Piel & 14280 & 4851 & 7 \\
\hline Sockect & 14033 & 4753 & 8 \\
\hline Grasa & 116494 & 26515 & 4 \\
\hline Músculo & 90995 & 19899 & 4 \\
\hline
\end{tabular}

Fuente. Elaboración propia.

\section{RESULTADOS}

En este estudio se hizo la comparación de la distribución y concentración de deformaciones, esfuerzos a compresión, y esfuerzo de von Mises debido a las condiciones de carga. Se encontró que la mayor concentración de esfuerzos y deformaciones se presenta en el tejido muscular en la zona distal del hueso residual, como se muestra en la Figura 7. Los máximos esfuerzos y deformaciones ocurren durante la fase de calzado del socket, que corresponden a los primeros diez segundos del análisis según se muestra en la Figura 7. Cada uno de

Tabla 2. Condiciones de carga en el modelo.

\begin{tabular}{|l|c|c|l|l|l|l|}
\hline Condición de Carga & $\mathrm{Rz}[\mathrm{N}]$ & $\mathrm{Ry}[\mathrm{N}]$ & $\mathrm{Rx}[\mathrm{N}]$ & $\mathrm{Mx}[\mathrm{N} . \mathrm{m}]$ & $\mathrm{My}[\mathrm{N} . \mathrm{m}]$ & $\mathrm{Mz}[\mathrm{N} . \mathrm{m}]$ \\
\hline Monopedestación (marcha normal) & 961 & 352 & 12.2 & 167 & 3.87 & 2.18 \\
\hline Bipedestación & 480.5 & 176 & 0 & 83.5 & 0 & 0 \\
\hline Monopedastición por Escaleras & 4805 & 1760 & 61 & 835 & 19.35 & 10.9 \\
\hline
\end{tabular}

Fuente. Elaboración propia.

\subsection{Mallado}

Después de tener definida la geometría, las propiedades mecánicas y las condiciones de borde, se realizó el mallado del dominio del problema, en este caso, debido a la complejidad del fémur, se emplearon elementos tetraédricos, y para los demás elementos se utilizaron elementos hexaedrales. Los datos de número de nodos y el tamaño y número elementos empleados en los modelos se encuentran en la Tabla 3. los esfuerzos se ven afectados en la fase de apoyo de la marcha sin superar el umbral de muerte celular (ver Figura 7 (a)). Las deformaciones presentan una reducción del $10 \%$ en la fase de relajación en los tres segundos posteriores a la fase desplazamiento y un aumento del 2 $\%$ asociado a la fase de apoyo de la marcha (ver . Figura 7 (b)). Lo que indica que la mayor deformación y esfuerzo a compresión está asociada al momento en que la persona se calza la prótesis y se ven afectados por cada paso que da la persona. 


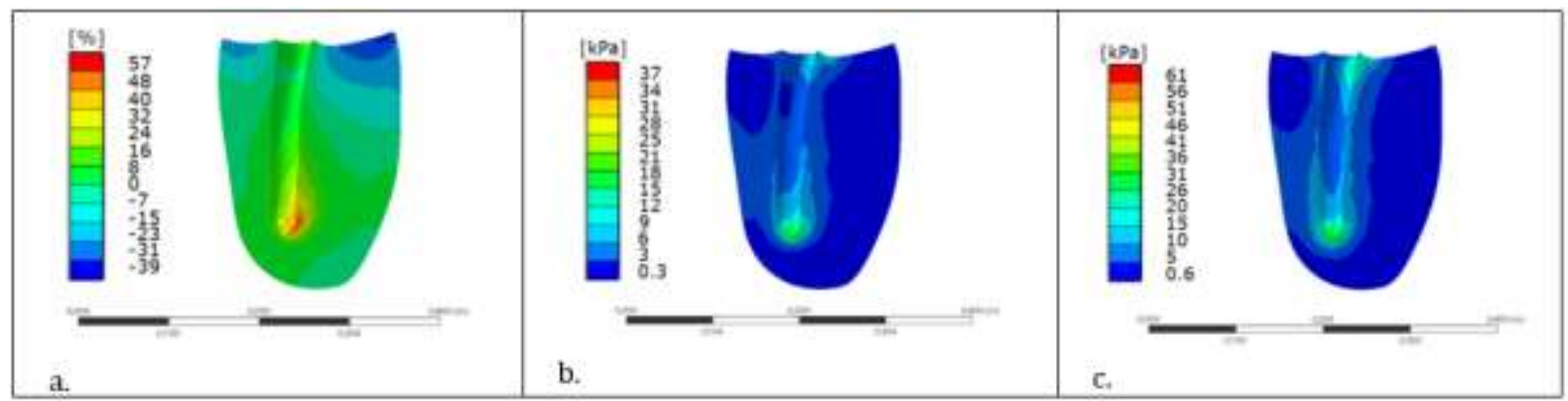

Figura 6. Distribución de (a). Deformación (b). Esfuerzos a compresión (c). Esfuerzo Von Mises, en el tejido muscular. Fuente. Elaboración propia.

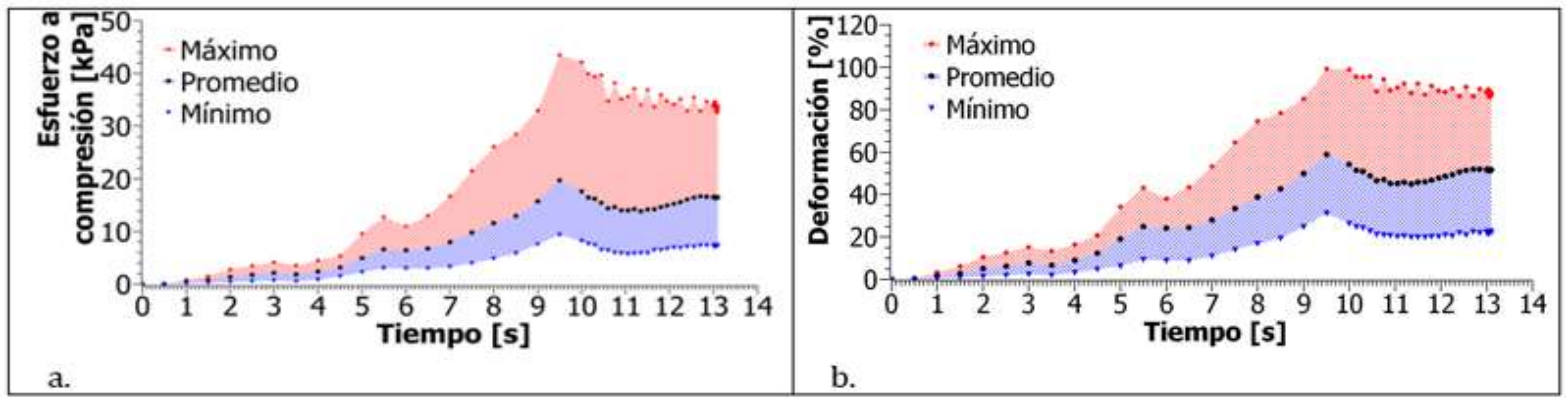

Figura 7. (a). Esfuerzo a compresión máximo, mínimo y promedio, (b). Deformación máxima, mínima y promedio en el músculo en la zona distal del fémur. Fuente. Elaboración propia.

En la Figura 8, Figura 9 y Figura 10, se hace la comparación de las deformaciones, esfuerzos a compresión y esfuerzos de von Mises respectivamente en diferentes condiciones de carga; bipedestación que corresponde al apoyo de una persona sobre la pierna sana y la prótesis; monopedestación condición donde la persona está en la fase de apoyo de la marcha con la prótesis y la fase de apoyo correspondiente al momento en que una persona está subiendo escaleras o una superficie inclinada.

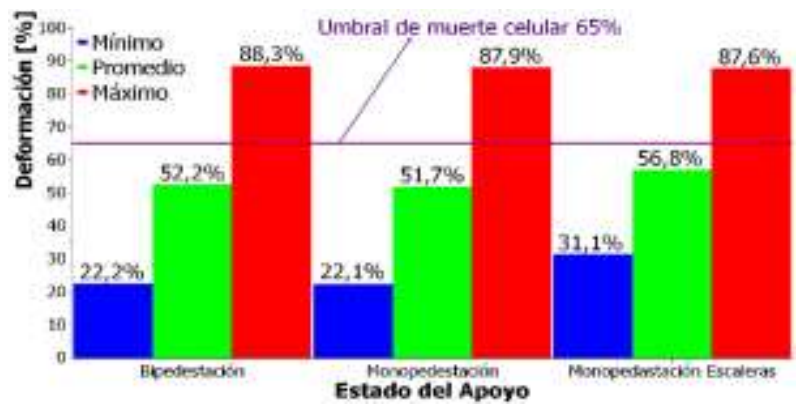

Figura 8. Deformaciones máximas, mínimas y promedio debido a las condiciones de carga. Fuente. Elaboración propia.
En la Figura 8 se muestran las deformaciones que se alcanzan en el instante en que la persona se encuentra en cada condición de carga (bipedestación, monopedestación y monopedestación en escaleras) donde se obtiene que las deformaciones desarrolladas en bipedestación y monopedestación, tienen una variación mínima de $0.1 \%$, y una máxima de $0.5 \%$ para los valores mínimos y promedios respectivamente. En comparación con la fase de apoyo en pendientes o escaleras, se evidencia una variación del $9 \%$ para las deformaciones mínimas, mientras que para los valores promedios de deformación hay un cambio del $5.6 \%$. Las deformaciones máximas se encuentran en el mismo margen con diferencias entre $0.3 \%$ y $0.4 \%$ sobrepasando los valores de umbral de muerte celular de $65 \%$, con valores cercanos al $88 \%$ de deformación a compresión. A pesar que existe una variación en la magnitud de las deformaciones desarrolladas por las diferentes condiciones de carga, no hay un cambio significativo por el tipo de condición de carga cuando es aplicada durante 0.1 segundo. 


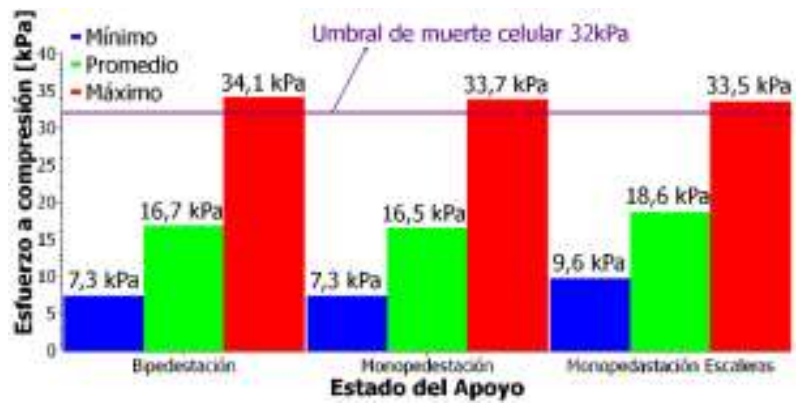

Figura 9. Esfuerzos a compresión máximos, mínimos y promedio debido a las condiciones de carga. Fuente. Elaboración propia.

Los resultados de esfuerzos a compresión se muestran en la Figura 9, donde los valores máximos de esfuerzo están cerca de $34 \mathrm{kPa}$ con una diferencia del $0.4 \mathrm{kPa}$ estando sobre el umbral de muerte celular de $32 \mathrm{kPa}$, y donde se presenta la mayor variación de $2.3 \mathrm{kPa}$ y $1.9 \mathrm{kPa}$ en los esfuerzos a compresión mínimos y promedios respectivamente. Para los esfuerzos de von Mises, se encuentra una variación máxima de $5.2 \mathrm{kPa}$ en los esfuerzos mínimos desarrollados. al igual que la tendencia de las deformaciones desarrolladas por el tipo de marcha, los esfuerzos a comprensión (ver Figura 9) y de von Mises (ver Figura 10), no presentan un cambio significativo, pero hay zonas donde se desarrollan esfuerzos y deformaciones que están por encima del umbral de muerte celular, como es el caso de los esfuerzos y deformaciones máximos, donde estos no presentan una variación dependiente del tipo de marcha o condición de carga.

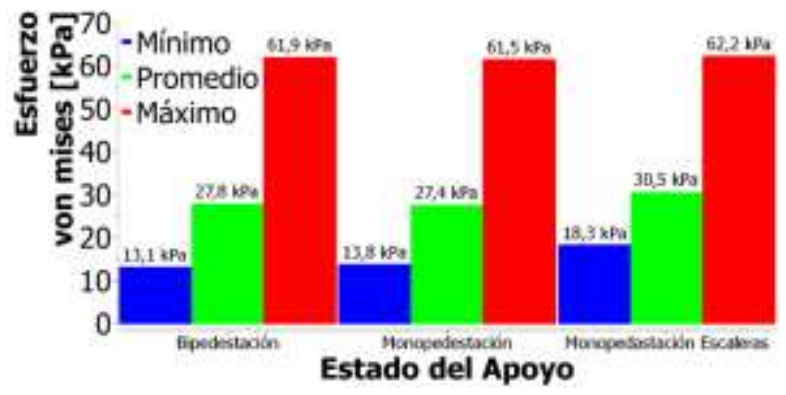

Figura 10. Esfuerzos von Mises máximos, mínimos y promedio debido a las condiciones de carga. Fuente. Elaboración propia.

\section{CONCLUSIONES}

Las úlceras por presión son complicaciones que se desarrollan de forma diferente de una persona a otra, donde influyen principalmente las condiciones demográficas de las personas y las enfermedades que padezcan; siendo más propensas a sufrir una úlcera por presión personas que padecen diabetes o enfermedades cardiovasculares. Las úlceras por presión más críticas son las que se desarrollan en el tejido interno; principalmente el tejido muscular cuando se encuentran bajo la acción de presiones superiores a $32 \mathrm{kPa}$ y deformaciones mayores al $65 \%$, que son los valores del umbral de muerte celular en tiempos de exposición menores a una hora [5], [6]. Estos umbrales son un punto de referencia, y pese a que no corresponden a mediciones en tejido humano, proporcionan una buena aproximación al comportamiento real de los tejidos.

Las condiciones antropométricas utilizadas para desarrollar el modelo 3D del miembro residual, permiten tener una aproximación al comportamiento real de un amputado transfemoral. Se encontró que la distribución y concentración de los esfuerzos y deformaciones críticos para desarrollar una úlcera por presión interna o una herida en el tejido interno, se localizan en el tejido muscular cerca de la zona distal del fémur residual; donde se transmiten los esfuerzos y deformaciones por la acción de la prominencia ósea, estos resultados concuerdan con los reportados por Portnoy et al. [36] y Gefen et al. [11] en sus investigaciones, permitiendo validar los modelos desarrollados bajo las condiciones geométricas propuestas y el uso de un modelo multicapa compuesto de materiales hiperelásticos.

Dado que el tiempo de aplicación de las condiciones de carga fue muy corto, se ve que no hay un valor representativo que permita establecer que solo por las reacciones desarrolladas en la posición de bipedestación, monopedestación en marcha normal o por superficies inclinadas o escaleras, se generen esfuerzos y deformaciones que puedan desarrollar heridas en el tejido interno, sin embargo, se determinó que los mayores esfuerzos y deformaciones en el tejido interno muscular se presentan durante la fase de calzado de la prótesis. No obstante, cabe anotar que cuando la persona se encuentra caminando por superficies inclinadas o escaleras, hay un aumento de los esfuerzos y deformaciones presentes en el tejido muscular, sin presentar una variación importante cuando se encuentra en la fase de apoyo de la marcha normal y en bipedestación. De los resultados obtenidos se tienen valores de deformación promedio cercanos a $50 \%$, (ver Figura 7 (b) y Figura 8), de acuerdo a los resultados obtenidos por Breuls et al. [40], cuando se tienen deformaciones de esta magnitud hay daño en el tejido que está cerca a una prominencia osea y este daño se evidencia alrededor de dos horas de mantener la deformación, si aumenta la magnitud de las deformaciones pesentes en los tejidos, el tiempo en el que se genera una ulcera por presión o una herida interna se 
reduce. La magnitud de los esfuerzos a compresión que se tiene en el tejido interno son cercanos a $20 \mathrm{kPa}$ y alcanzan valores maximos de $34 \mathrm{kPa}$, de acuerdo a los resultados obtenidos por linder et al. [41], cuando se tiene estos esfuerzos durante tiempos mayores a 90 minutos, se puede desarrollar una herida en el tejidos internos. Siendo entonces el tiempo de generación de una herida interna, función de la magnitud de los esfuerzos y deformaciones que se desarrollan en el tejido interno.

Para predecir el daño en los tejidos blandos y como se propagan las heridas en el tejido; no es suficiente con determinar umbrales de esfuerzo y deformación, es necesario tener en cuenta el tiempo de aplicación de las cargas ya que se evidenció en los resultados que, tiempos cortos no reflejan una influencia de las condiciones de carga, por lo que es necesario para futuras investigaciones emplear modelos viscoelásticos donde se pueda asociar la variable tiempo.

\section{REFERENCIAS}

[1] Dan R. Berlowitz y Van B. Wilking, «Risk Factors for Pressure Sores, A Comparison of Cross-Sectional And Cohort-Derive Data», pp. 1043-1050, 1989.

[2] D. Byrne y C. Salzberg, «Major risk factors for pressure ulcers in the spinal cord disabled: a literature review - Major-risk-factors-for-pressure-ulcers-in-thespinal-cord-disabled-A-literature-review.pdf», pp. 255263, 1996.

[3] Leon Bennett y Bok Y. Lee, «Vertical shear existence in anaimal pressure threshold experiments», pp. 18-24, 1988.

[4] Baldwin y Kathleen M., «Transcutaneous Oximetry and Skin Surface Temperature as Objective Measures of Pressuere Ulcer Risk», LWW, 2001.

[5] E. Linder-Ganz, S. Engelberg, M. Scheinowitz, y A. Gefen, «Pressure-time cell death threshold for albino rat skeletal muscles as related to pressure sore biomechanics», J. Biomech., vol. 39, n. ${ }^{\circ}$ 14, pp. 27252732, 2006.

[6] A. Gefen, B. van Nierop, D. L. Bader, y C. W. Oomens, «Strain-time cell-death threshold for skeletal muscle in a tissue-engineered model system for deep tissue injury», J. Biomech., vol. 41, n. ${ }^{\circ}$ 9, pp. 2003-2012, 2008.

[7] R. Á. Chaurand, L. R. P. León, y E. L. G. Muñoz, Dimensiones antropométricas de población latinoamericana. Universidad de Guadalajara, Centro Universitario de Arte, Arquitectura y Diseño, División de
Tecnología y Procesos, Departamento de Producción y Desarrollo, Centro de Investigaciones en Ergonomía, 2001.

[8] C. A. Mejía y J. A. Villarraga, «Influencia de las condiciones de carga, porcentaje de grasa del muñón y longitud del hueso residual en la generación de úlceras por presión en amputados transfemorales», Trabajo de fin de máster, Universidad de Antioquia, Medellín, 2017.

[9] J. Díaz y O. Espinoza-Navarro, «Determinación del Porcentaje de Masa Grasa, según Mediciones de Perímetros Corporales, Peso y Talla: Un Estudio de Validación», Int. J. Morphol., vol. 30, n. ${ }^{\circ} 4$, pp. 16041610, dic. 2012.

$[10] \ll$ Predicting Pressure Distribution Between Transfemoral Prosthetic Socket and Residual Limb Using Finite Element Analysis - viewcontent.cgi».

[11]R. Sopher, J. Nixon, C. Gorecki, y A. Gefen, «Exposure to internal muscle tissue loads under the ischial tuberosities during sitting is elevated at abnormally high or low body mass indices», J. Biomech., vol. 43, n. ${ }^{\circ}$ 2, pp. 280-286, 2010.

[12]W. C. C. Lee, M. Zhang, X. Jia, y J. T. M. Cheung, «Finite element modeling of the contact interface between trans-tibial residual limb and prosthetic socket», Med. Eng. Phys., vol. 26, n. ${ }^{\circ}$ 8, pp. 655-662, oct. 2004.

[13]M. Zhang y A. F. Mak, «A finite element analysis of the load transfer between an above-knee residual limb and its prosthetic socket--roles of interface friction and distal-end boundary conditions», IEEE Trans. Rehabil. Eng. Publ. IEEE Eng. Med. Biol. Soc., vol. 4, n. ${ }^{\circ}$ 4, pp. 337-346, dic. 1996.

[14]M. Zhang y C. Roberts, «Comparison of computational analysis with clinical measurement of stresses on below-knee residual limb in a prosthetic socket», Med. Eng. Phys., vol. 22, n. ${ }^{\circ}$ 9, pp. 607-612, nov. 2000.

[15]G. Boyer, J. Molimard, M. Ben Tkaya, H. Zahouani, M. Pericoi, y S. Avril, «Assessment of the in-plane biomechanical properties of human skin using a finite element model updating approach combined with an optical full-field measurement on a new tensile device», J. Mech. Behav. Biomed. Mater., vol. 27, pp. 273-282, nov. 2013.

[16]A. Delalleau, G. Josse, J.-M. Lagarde, H. Zahouani, y J.-M. Bergheau, «A nonlinear elastic behavior to identify the mechanical parameters of human skin in 
vivo», Skin Res. Technol., vol. 14, n. ${ }^{\circ} 2$, pp. 152-164, 2008.

[17]A. . Delalleau, G. . Josse, J.-M. . Lagarde, H. . Zahouani, J.-M. . Bergheau, y R. . Toscano, «A new stochastic inverse identification of the mechanical properties of human skin», Eng. Optim., vol. 43, n. ${ }^{\circ}$ 1, pp. 61-75, 2011.

[18]C. Flynn, A. Taberner, y P. Nielsen, «Mechanical characterisation of in vivo human skin using a $3 \mathrm{D}$ forcesensitive micro-robot and finite element analysis», Biomech. Model. Mechanobiol., vol. 10, n. ${ }^{\circ}$ 1, pp. 27-38, feb. 2011.

[19]C. Flynn, A. Taberner, y P. Nielsen, «Modeling the Mechanical Response of In Vivo Human Skin Under a Rich Set of Deformations», Ann. Biomed. Eng., vol. 39, n. ${ }^{\circ}$ 7, pp. 1935-1946, jul. 2011.

[20]R. B. . Groves, S. A. . Coulman, J. C. . Birchall, y S. L. . Evans, "Quantifying the mechanical properties of human skin to optimise future microneedle device design», Comput. Methods Biomech. Biomed. Engin., vol. 15, n. ${ }^{\circ}$ 1, pp. 73-82, 2012.

[21]F. M. Hendriks, D. Brokken, J. Van Eemeren, C. W. J. Oomens, F. P. T. Baaijens, y J. Horsten, «A numericalexperimental method to characterize the non-linear mechanical behaviour of human skin», Skin Res. Technol., vol. 9, n. ${ }^{\circ} 3$, pp. 274-283, 2003.

[22]F. M. Hendriks, D. Brokken, C. W. J. Oomens, y F. P. T. Baaijens, «Influence of hydration and experimental length scale on the mechanical response of human skin in vivo, using optical coherence tomography», Skin Res. Technol. Off. J. Int. Soc. Bioeng. Skin ISBS Int. Soc. Digit. Imaging Skin ISDIS Int. Soc. Skin Imaging ISSI, vol. 10, n. ${ }^{\circ}$ 4, pp. 231-241, nov. 2004.

[23]F. M. Hendriks, D. Brokken, C. W. J. Oomens, D. L. Bader, y F. P. T. Baaijens, «The relative contributions of different skin layers to the mechanical behavior of human skin in vivo using suction experiments», Med. Eng. Phys., vol. 28, n. ${ }^{\circ}$ 3, pp. 259-266, abr. 2006.

[24]F. Khatyr, C. Imberdis, P. Vescovo, D. Varchon, y J.-M. Lagarde, «Model of the viscoelastic behaviour of skin in vivo and study of anisotropy», Skin Res. Technol., vol. 10, n. ${ }^{\circ} 2$, pp. 96-103, 2004.

[25]J. T. Iivarinen, R. K. Korhonen, P. Julkunen, y J. S. Jurvelin, «Experimental and computational analysis of soft tissue stiffness in forearm using a manual indentation device», Med. Eng. Phys., vol. 33, n. ${ }^{\circ}$ 10, pp. 1245-1253, dic. 2011.

[26]N. F. A. Manan et al., «Determining hyperelastic parameters of human skin using 2D finite element modelling and simulation», presentado en 2012 IEEE Symposium on Humanities, Science and Engineering Research (SHUSER), 2012, pp. 805-809.

[27]J. Mahmud, C. Holt, S. Evans, N. F. A. Manan, y M. Chizari, «A Parametric Study and Simulations in Quantifying Human Skin Hyperelastic Parameters», Procedia Eng., vol. 41, pp. 1580-1586, 2012.

[28]H. V. Tran, F. Charleux, M. Rachik, A. Ehrlacher, y M. C. Ho Ba Tho, «In vivo characterization of the mechanical properties of human skin derived from MRI and indentation techniques», Comput. Methods Biomech. Biomed. Engin., vol. 10, n. ${ }^{\circ}$ 6, pp. 401-407, dic. 2007.

[29]R. J. . Lapeer, P. D. . Gasson, y V. . Karri, «A hyperelastic finite-element model of human skin for interactive real-time surgical simulation», IEEE Trans. Biomed. Eng., vol. 58, n. ${ }^{\circ}$ 4, pp. 1013-1022, 2011.

[30]S. Portnoy, I. Siev-Ner, Z. Yizhar, A. Kristal, N. Shabshin, y A. Gefen, «Surgical and Morphological Factors that Affect Internal Mechanical Loads in Soft Tissues of the Transtibial Residuum», Ann. Biomed. Eng., vol. 37, n. ${ }^{\circ}$ 12, pp. 2583-2605, sep. 2009.

[31]R. O. Potts, D. A. Chrisman Jr., y E. M. Buras Jr., «The dynamic mechanical properties of human skin in vivo», J. Biomech., vol. 16, n. ${ }^{\circ}$ 6, pp. 365-372, 1983.

[32]L. Duchemin et al., «Prediction of mechanical properties of cortical bone by quantitative computed tomography», Med. Eng. Phys., vol. 30, n. ${ }^{\circ} 3$, pp. 321328, abr. 2008.

[33]S. Portnoy et al., «Real-time patient-specific finite element analysis of internal stresses in the soft tissues of a residual limb: a new tool for prosthetic fitting», Ann. Biomed. Eng., vol. 35, n. ${ }^{\circ}$ 1, pp. 120-135, ene. 2007.

[34]S. Portnoy et al., «Internal mechanical conditions in the soft tissues of a residual limb of a trans-tibial amputee», J. Biomech., vol. 41, n. ${ }^{\circ}$ 9, pp. 1897-1909, 2008.

[35]X. Jia, M. Zhang, X. Li, y W. C. C. Lee, «A quasidynamic nonlinear finite element model to investigate prosthetic interface stresses during walking for trans- 
tibial amputees», Clin. Biomech., vol. 20, n. ${ }^{\circ}$ 6, pp. 630635, jul. 2005.

[36]S. Portnoy, I. Siev-Ner, N. Shabshin, A. Kristal, Z. Yizhar, y A. Gefen, «Patient-specific analyses of deep tissue loads post transtibial amputation in residual limbs of multiple prosthetic users», J. Biomech., vol. 42, n. ${ }^{\circ} 16$, pp. 2686-2693, dic. 2009.

[37]M. J. Adams, B. J. Briscoe, y S. A. Johnson, «Friction and lubrication of human skin», Tribol. Lett., vol. 26, n. ${ }^{\circ}$ 3, pp. 239-253, mar. 2007.

[38]J. M. Sanders, J. M. Greve, S. B. Mitchell, y S. G. Zachariah, «Material properties of commonly-used interface materials and their static coefficients of friction with skin and socks».

[39]Vanessa Restrepo, Junes Villarraga, y Jaime Velez, «Surface optimization of a socket for a transfemoral amputee that reduces the stresses varying the friction coefficient», vol. 1, 2014.

[40]R. G. M. Breuls, C. V. C. Bouten, C. W. J. Oomens, D. L. Bader, y F. P. T. Baaijens, «A Theoretical Analysis of Damage Evolution in Skeletal Muscle Tissue With Reference to Pressure Ulcer Development», J. Biomech. Eng., vol. 125, n. ${ }^{\circ}$ 6, pp. 902-909, dic. 2003.

[41]E. Linder-Ganz y A. Gefen, «Stress Analyses Coupled With Damage Laws to Determine Biomechanical Risk Factors for Deep Tissue Injury During Sitting», J. Biomech. Eng., vol. 131, n. ${ }^{\circ}$ 1, pp. 011003-011003-13, nov. 2008. 